\title{
Equivalent Conditions of Complete $p$ th Moment Convergence for Weighted Sums of I. I. D. Random Variables under Sublinear Expectations
}

\author{
Mingzhou Xu (i) and Kun Cheng \\ School of Information Engineering, Jingdezhen Ceramic University, Jingdezhen 333403, China \\ Correspondence should be addressed to Mingzhou Xu; mingzhouxu@whu.edu.cn
}

Received 20 July 2021; Revised 19 September 2021; Accepted 30 September 2021; Published 18 October 2021

Academic Editor: Ya Jia

Copyright (C) 2021 Mingzhou Xu and Kun Cheng. This is an open access article distributed under the Creative Commons Attribution License, which permits unrestricted use, distribution, and reproduction in any medium, provided the original work is properly cited.

We investigate the complete pth moment convergence for weighted sums of independent, identically distributed random variables under sublinear expectations space. Using moment inequality and truncation methods, we prove the equivalent conditions of complete $p$ th moment convergence of weighted sums of independent, identically distributed random variables under sublinear expectations space, which complement the corresponding results obtained in Guo and Shan (2020).

\section{Introduction}

Peng $[1,2]$ presented the concept of the sublinear expectation space to study the uncertainty of probability and distribution. The seminal work of Peng $[1,2]$ encourages people to study limit theorems under sublinear expectations space. Zhang [3-5] proved results including exponential inequalities, Rosenthal's inequalities, and Donsker's invariance principle under sublinear expectations. Wu [6] obtained precise asymptotics for complete integral convergence. $\mathrm{Xu}$ and Cheng [7] studied precise asymptotics in the law of iterated logarithm under sublinear expectations. The interested reader could refer to Xu and Zhang [8, 9], Chen [10], Gao and Xu [11], Fang et al. [12], Hu et al. [13], $\mathrm{Hu}$ and Yang [14], Huang and $\mathrm{Wu}$ [15], Kuczmaszewska [16], $\mathrm{Ma}$ and $\mathrm{Wu}$ [17], Wang and $\mathrm{Wu}$ [18], $\mathrm{Wu}$ and Jiang [19], $\mathrm{Yu}$ and $\mathrm{Wu}[20]$, Zhang [21], Zhong and $\mathrm{Wu}$ [22], and references therein for more limit theorems under sublinear expectations.

Recently, Guo and Shan [23] studied equivalent conditions of complete $q$ th moment convergence for weighted sums of sequences of negatively orthant dependent random variables. Xu and Cheng [24] obtained equivalent conditions of complete convergence for weighted sums of sequences of i. i. d. random variables under sublinear expectations. Motivated by the work of Guo and Shan [23] and $\mathrm{Xu}$ and Cheng [24], here we try to prove the equivalent conditions of complete $p$ th moment convergence of weighted sums of independent, identically distributed random variables under sublinear expectations space, which complement the corresponding results obtained in Guo and Shan [23], also extend results in $\mathrm{Xu}$ and Cheng [24] from complete convergence to complete $p$ th moment convergence.

We organized the rest of this paper as follows. In Section 2 , we give necessary basic notions, concepts, and relevant properties and present necessary lemmas under sublinear expectations. In Section 3, we give our main results, Theorems 1-4, whose proofs are presented in Section 4.

\section{Preliminaries}

As in $\mathrm{Xu}$ and Cheng [24], we adopt similar notations as in the work by Peng [2] and Chen [10]. Suppose that $(\Omega, \mathscr{F})$ is a given measurable space. We assume that $\mathscr{H}$ is a subset of all random variables on $(\Omega, \mathscr{F})$ such that $I_{A} \in \mathscr{H}$ (cf. [10]), where $A \in \mathscr{F}$, and $X_{1}, \ldots, X_{n} \in \mathscr{H}$ implies $\varphi\left(X_{1}, \ldots, X_{n}\right)$ $\in \mathscr{H}$, for each $\varphi \in \mathscr{C}_{l \text {,Lip }}\left(\mathbb{R}^{n}\right)$, where $\mathscr{C}_{l, \text { Lip }}\left(\mathbb{R}^{n}\right)$ denotes the linear space of (local Lipschitz) function $\varphi$ satisfying 
$|\varphi(\mathbf{x})-\varphi(\mathbf{y})| \leq C\left(1+|\mathbf{x}|^{m}+|\mathbf{y}|^{m}\right)(|\mathbf{x}-\mathbf{y}|), \quad \forall \mathbf{x}, \mathbf{y} \in \mathbb{R}^{n}$,

for some $C>0$ and $m \in \mathbb{N}$ depending on $\varphi$.

Definition 1. A sublinear expectation $\mathbb{E}$ on $\mathscr{H}$ is a functional $\mathbb{E}: \mathscr{H} \mapsto \overline{\mathbb{R}}:=[-\infty, \infty]$ satisfying the following properties: for all $X, Y \in \mathscr{H}$, we have

(a) Monotonicity: if $X \geq Y$, then $\mathbb{E}[X] \geq \mathbb{E}[Y]$

(b) Constant preserving: $\mathbb{E}[c]=c, \forall c \in \mathbb{R}$

(c) Positive homogeneity: $\mathbb{E}[\lambda X]=\lambda \mathbb{E}[X], \forall \lambda \geq 0$

(d) Subadditivity: $\mathbb{E}[X+Y] \leq \mathbb{E}[X]+\mathbb{E}[Y]$ whenever $\mathbb{E}[X]+\mathbb{E}[Y]$ is not of the form $\infty-\infty$ or $-\infty+\infty$ obeys

A set function $V: \mathscr{F} \mapsto[0,1]$ is said to be a capacity if it

(a) $V(\varnothing)=0$ and $V(\Omega)=1$.

(b) $V(A) \leq V(B), A \subset B$ and $A, B \in \mathscr{F}$.

Moreover, if $V$ is continuous, then $V$ should satisfy

(c) $V\left(A_{n}\right) \uparrow V(A)$ if $A_{n} \uparrow A$.

(d) $V\left(A_{n}\right) \downarrow V(A)$ if $A_{n} \downarrow A$.

A capacity $V$ is called subadditive if $V(A+B) \leq V(A)+V(B), A, B \in \mathscr{F}$.

In this paper, given a sublinear expectation space $(\Omega, \mathscr{H}, \mathbb{E})$, set $\mathbb{V}(A):=\inf \left\{\mathbb{E}[\xi]: I_{A} \leq \xi, \xi \in \mathscr{H}\right\}=\mathbb{E}\left[I_{A}\right]$, $\forall A \in \mathscr{F}$ (see (8) and the definitions of $\mathbb{V}$ above (8) in [4]). Clearly, $\mathbb{V}$ is a subadditive capacity. Denote the Choquet expectations $C_{\mathbb{V}}$ by

$$
C_{\mathbb{V}}(X):=\int_{0}^{\infty} \mathbb{V}(X>x) \mathrm{d} x+\int_{-\infty}^{0}(\mathbb{V}(X>x)-1) \mathrm{d} x .
$$

Suppose that $\mathbf{X}=\left(X_{1}, \ldots, X_{m}\right), \quad X_{i} \in \mathscr{H}, \quad$ and $\mathbf{Y}=\left(Y_{1}, \ldots, Y_{n}\right), Y_{i} \in \mathscr{H}$, are two random vectors on $(\Omega, \mathscr{H}, \mathbb{E}) . \mathbf{Y}$ is called to be independent of $\mathbf{X}$ if, for each Borel-measurable function $\psi$ on $\mathbb{R}^{m} \times \mathbb{R}^{n}$ with $\psi(\mathbf{X}, \mathbf{Y}), \psi(\mathbf{x}, \mathbf{Y}) \in \mathscr{H} ; \quad$ for each $\mathbf{x} \in \mathbb{R}^{m}$, we have $\mathbb{E}[\psi(\mathbf{X}, \mathbf{Y})]=\mathbb{E}\left[\left.\mathbb{E} \psi(\mathbf{x}, \mathbf{Y})\right|_{\mathbf{X}=\mathbf{X}}\right] \quad$ whenever $\bar{\psi}(\mathbf{x}):=\mathbb{E}[|\psi(\mathbf{x}, \mathbf{Y})|]<\infty$ for each $\mathbf{x}$ and $\mathbb{E}[|\bar{\psi}(\mathbf{X})|]<\infty$ (see Definition 2.5 in [10]). $\left\{X_{n}\right\}_{n=1}^{\infty}$ is called a sequence of independent random variables if $X_{n+1}$ is independent of $\left(X_{1}, \ldots, X_{n}\right)$, for each $n \geq 1$.

Assume that $\mathbf{X}_{1}$ and $\mathbf{X}_{2}$ are two $n$-dimensional random vectors defined, respectively, in sublinear expectation spaces $\left(\Omega_{1}, \mathscr{H}_{1}, \mathbb{E}_{1}\right)$ and $\left(\Omega_{2}, \mathscr{H}_{2}, \mathbb{E}_{2}\right)$. They are called identically distributed if, for every Borel-measurable function $\psi$ such that $\psi\left(\mathbf{X}_{1}\right) \in \mathscr{H}_{1}, \psi\left(\mathbf{X}_{2}\right) \in \mathscr{H}_{2}$,

$$
\mathbb{E}_{1}\left[\psi\left(\mathbf{X}_{1}\right)\right]=\mathbb{E}_{2}\left[\psi\left(\mathbf{X}_{2}\right)\right],
$$

whenever the sublinear expectations are finite. $\left\{X_{n}\right\}_{n=1}^{\infty}$ is called to be identically distributed if, for each $i \geq 1, X_{i}$ and $X_{1}$ are identically distributed.

In the sequel, we suppose that $\mathbb{E}$ is countably subadditive, i.e., $\mathbb{E}(X) \leq \sum_{n=1}^{\infty} \mathbb{E}\left(X_{n}\right)$, whenever $X \leq \sum_{n=1}^{\infty} X_{n}, X, X_{n}$ $\in \mathscr{H}$, and $X \geq 0, X_{n} \geq 0, n=1,2, \ldots$. Let $C$ denote a positive constant which may differ from line to line. $I(A)$ or $I_{A}$ represents the indicator function of $A, a_{n} \ll b_{n}$ means that there exists a constant $C>0$ such that $a_{n} \leq C b_{n}$ for $n$ large sufficiently, and $a_{n} \approx b_{n}$ means that $a_{n} \ll b_{n}$ and $b_{n} \ll a_{n}$. We use $\log x$ for $\ln \max \{e, x\}$.

We first present several necessary lemmas to prove our main results. By using Corollary 2.2, Theorem 2.3 in [5], the proofs of Theorem 3 in [25], and Minkowski's inequality under sublinear expectations, we see that the following lemma holds.

Lemma 1 (cf. Lemma 2.3 in [24]). Suppose that $\left\{X_{i}, 1 \leq i \leq n\right\}$ is a sequence of independent random variables under sublinear expectation space $(\Omega, \mathscr{H}, \mathbb{E})$ with $\mathbb{E}\left[X_{i}\right] \leq 0$, $\mathbb{E}\left|X_{i}\right|^{M}<\infty, 1 \leq i \leq n$, and $M \geq 2$. Then,

$$
\mathbb{E} \max _{1 \leq j \leq n}\left|\sum_{i=1}^{j} X_{i}\right|^{M} \leq C \log ^{M} n\left(\sum_{i=1}^{n} \mathbb{E}\left|X_{i}\right|^{M}+\left(\sum_{i=1}^{n} \mathbb{E}\left|X_{i}\right|^{2}\right)^{M / 2}\right),
$$

where $C$ depends on $M$ only.

Proof. For readers' convenience, we give complete proofs here. First, for $b \geq 0$ and $n \geq 1$, set $S_{b, n}=\sum_{k=b+1}^{b+n} X_{i}$ and $L_{b, n}=\max _{1 \leq k \leq n}\left|S_{b, k}\right|$, and

$$
g\left(F_{b, n}\right)=C_{M}\left(\sum_{i=b+1}^{b+n} \mathbb{E}\left|X_{i}\right|^{M}+\left(\sum_{i=b+1}^{b+n} \mathbb{E}\left|X_{i}\right|^{2}\right)^{M / 2}\right),
$$

where $C_{M}$ depends on $M$ determined as in (2.8) of Corollary 2.2 (b) in [5]. By Corollary 2.2 in [5], we know that, for all $b \geq 0$ and $n \geq 1$,

$$
\mathbb{E}\left(\left|S_{b, n}\right|^{M}\right) \leq g\left(F_{b, n}\right) .
$$

Obviously, for $b \geq 0$ and $1 \leq k \leq k+l$,

$$
g\left(F_{b, k}\right)+g\left(F_{b+k, l}\right) \leq g\left(F_{b, k+l}\right) .
$$

Set $\Lambda(1)=1$, and for $n \geq 2, \Lambda(n)=1+\Lambda(m-1)$, where $m$ is the integer part of $(1 / 2)(n+2)$. $1+\log (2(m-1)) \leq \log (2 n)$ implies that $\Lambda(n) \leq \log (2 n)$. Now, it is enough to prove that, for $b \geq 0$ and $n \geq 1$,

$$
\mathbb{E}\left(L_{b, n}^{M}\right) \leq(\Lambda(n))^{M} g\left(F_{b, n}\right) .
$$

As in the proof of Theorem 4 of Móricz [25], let $n>1$ be given. Obviously, $n=2 m-1$ or $2 m-2$. For $b \geq 0$ and $m \leq k \leq n$, we see that

$$
\left|S_{b, k}\right| \leq\left|S_{b, m}\right|+\left|S_{b+m, k-m}\right|
$$

whence, for such $k$ 's,

$$
\left|S_{b, k}\right| \leq\left|S_{b, m}\right|+L_{b+m, n-m} .
$$

Since, for $1 \leq k<m$, we have $\left|S_{b, k}\right| \leq L_{b, m-1}$, hence, for $1 \leq k \leq n$,

$$
\left|S_{b, k}\right| \leq\left|S_{b, m}\right|+\left(L_{b, m-1}^{M}+L_{b+m, n-m}^{M}\right)^{1 / M}
$$

Thus, 


$$
L_{b, n} \leq\left|S_{b, m}\right|+\left(L_{b, m-1}^{M}+L_{b+m, n-m}^{M}\right)^{1 / M},
$$

and by Minkowski's inequality under sublinear expectations (see (4.10) in Proposition 4.2 of Chapter I of [2]),

$$
\left(\mathbb{E}\left(L_{b, n}^{M}\right)\right)^{1 / M} \leq\left(\mathbb{E}\left(\left|S_{b, m}^{M}\right|\right)\right)^{1 / M}+\left(\mathbb{E}\left(L_{b, m-1}^{M}\right)+\mathbb{E}\left(L_{b+m, n-m}^{M}\right)\right)^{1 / M} .
$$

Suppose now that (8) holds for $k<n$. Then, by the choice of $m$, we see that

$$
\begin{aligned}
\mathbb{E}\left(L_{b, m-1}^{M}\right) & \leq \Lambda^{M}(m-1) g\left(F_{b, m-1}\right), \\
\mathbb{E}\left(L_{b+m, n-m}^{M}\right) & \leq \Lambda^{M}(n-m) g\left(F_{b+m, n-m}\right) \\
& \leq \Lambda^{M}(m-1) g\left(F_{b+m, n-m}\right) .
\end{aligned}
$$

By these two inequalities above and (7), we see that

$$
\mathbb{E}\left(L_{b, m-1}^{M}\right)+\mathbb{E}\left(L_{b+m, n-m}^{M}\right) \leq \Lambda^{M}(m-1) g\left(F_{b, n}\right) .
$$

Finally, (6) implies

$$
\mathbb{E}\left(\left|S_{b, m}\right|^{M}\right) \leq g\left(F_{b, m}\right) \leq g\left(F_{b, n}\right) .
$$

By (13)-(16), we conclude that

$$
\left(\mathbb{E}\left(L_{b, n}^{M}\right)\right)^{1 / M} \leq(1+\Lambda(m-1)) g^{1 / M}\left(F_{b, n}\right)=\Lambda(n) g^{1 / M}\left(F_{b, n}\right),
$$

which implies the result. Hence, by (6), the conclusion of (8) is true for $n=1$. By induction, (8) holds for all $n=1,2, \ldots$. The proof is complete.

Lemma 2 (see Lemma 2.4 in $[24,26]$ ). Let $\left\{X_{n} ; n \geq 1\right\}$ be a sequence of independent random variables under sublinear expectation space $(\Omega, \mathscr{H}, \mathbb{E})$. Then, for all $n \geq 1$ and $x>0$,

$$
\left[1-\mathbb{V}\left(\max _{1 \leq j \leq n}\left|X_{j}\right|>x\right)\right]^{2} \sum_{j=1}^{n} \mathbb{V}\left(\left|X_{j}\right|>x\right) \leq 4 \mathbb{V}\left(\max _{1 \leq j \leq n}\left|X_{j}\right|>x\right) .
$$

Remark 1. In the proofs of Lemma 2.4 in $[24,26]$, by independence of $I\left(A_{k}\right):=I\left(\left|X_{k}\right|>x\right), k=1, \ldots, n$, $\mathbb{E}(X+c)=\mathbb{E}(c+X)=\mathbb{E}(X)+c$ for constant $c$ and $X \in \mathscr{H}$ (Definition 2.5 in [10]), we see that

$$
\begin{aligned}
\sum_{k=1}^{n} \mathbb{E}\left[I\left(A_{k}\right)\right] & =\sum_{k=1}^{n-2} \mathbb{E}\left[I\left(A_{k}\right)\right]+\mathbb{E}\left[I\left(A_{n-1}\right)+\mathbb{E}\left[I\left(A_{n}\right)\right]\right] \\
& =\sum_{k=1}^{n-2} \mathbb{E}\left[I\left(A_{k}\right)\right]+\mathbb{E}\left[\left.\left[x+\mathbb{E}\left[I\left(A_{n}\right)\right]\right]\right|_{x=I\left(A_{n-1}\right)}\right]=\sum_{k=1}^{n-2} \mathbb{E}\left[I\left(A_{k}\right)\right]+\mathbb{E}\left[\left.\mathbb{E}\left[x+I\left(A_{n}\right)\right]\right|_{\left.x=I\left(A_{n-1}\right)\right]}\right] \\
& =\sum_{k=1}^{n-2} \mathbb{E}\left[I\left(A_{k}\right)\right]+\mathbb{E}\left[I\left(A_{n-1}\right)+I\left(A_{n}\right)\right]=\cdots=\mathbb{E}\left[I\left(A_{1}\right)+\mathbb{E}\left[\sum_{k=2}^{n} I\left(A_{k}\right)\right]\right] \\
& =\mathbb{E}\left[\sum_{k=1}^{n} I\left(A_{k}\right)\right]
\end{aligned}
$$

which implies that Lemma 2 is valid. The difference between the sublinear expectations and linear expectations could be implied by the subtle observation that

$$
\begin{aligned}
& \mathbb{E}\left[I\left(A_{1}\right)-I\left(A_{2}\right)\right]=\mathbb{E}\left[\left.\left[\mathbb{E}\left[x-I\left(A_{2}\right)\right]\right]\right|_{x=I}\left(A_{1}\right)\right] \\
& =\mathbb{E}\left[\left.\left[x+\mathbb{E}\left[-I\left(A_{2}\right)\right]\right]\right|_{x=I\left(A_{1}\right)}\right]=\mathbb{E}\left[I\left(A_{1}\right)\right]+\mathbb{E}\left[-I\left(A_{2}\right)\right],
\end{aligned}
$$

which is not necessarily equal to $\mathbb{E}\left[I\left(A_{1}\right)\right]-\mathbb{E}\left[I\left(A_{2}\right)\right]$.

Lemma 3. Let $X$ be a random variable under sublinear expectation space $(\Omega, \mathscr{H}, \mathbb{E}), q>0, r>0$, and $p>0$. Then, the following are equivalent: (i)

$$
\begin{cases}C_{\mathbb{V}}\left(|X|^{p}\right)<\infty, & \text { for } p>\frac{r}{q} \\ C_{\mathbb{V}}\left(|X|^{r / q} \ln (|X|)\right)<\infty, & \text { for } p=\frac{r}{q} \\ C_{\mathbb{V}}\left(|X|^{r / q}\right)<\infty, & \text { for } p<\frac{r}{q}\end{cases}
$$

(ii)

$$
\int_{1}^{\infty} \mathrm{d} y \int_{1}^{\infty} y^{r-1} \mathbb{V}\left(|X|>x^{1 / p} y^{q}\right) \mathrm{d} x<\infty
$$


Proof

$$
\begin{aligned}
\int_{1}^{\infty} \mathrm{d} y \int_{1}^{\infty} y^{r-1} \mathbb{V}\left(|X|>x^{1 / p} y^{q}\right) \mathrm{d} x & =\int_{1}^{\infty} \mathrm{d} t \int_{t^{q}}^{\infty} t^{r-1} \mathbb{V}(|X|>s) s^{p-1} t^{-q p} \mathrm{~d} s\left(\text { Settings }=x^{1 / p} y^{q}, t=y\right) \\
& \approx \begin{cases}\int_{1}^{\infty} \mathbb{V}(|X|>s) s^{p-1} \mathrm{~d} s \approx \int_{1}^{\infty} \mathbb{V}(|X|>s) s^{p-1} \mathrm{~d} s \approx C_{\mathbb{V}}\left(|X|^{p}\right), & \text { for } p>\frac{r}{q}, \\
\int_{1}^{\infty} \mathbb{V}(|X|>s) s^{r / q-1} \ln (s) \mathrm{d} s \approx C_{\mathbb{V}}\left(|X|^{r / q} \ln (|X|)\right), & \text { for } p=\frac{r}{q}, \\
\int_{1}^{\infty} \mathbb{V}(|X|>s) s^{p-1+((r-p q) / q)} \mathrm{d} s \approx C_{\mathbb{V}}\left(|X|^{r / q}\right), & \text { for } p<\frac{r}{q}\end{cases}
\end{aligned}
$$

The proof is finished.

Lemma 4. Let $X$ be a random variable under sublinear expectation space $(\Omega, \mathscr{H}, \mathbb{E}), q>0, r>0$, and $p>0$. Then, the following is equivalent:

(i)

$$
\begin{cases}C_{\mathbb{V}}\left(|X|^{p}\right)<\infty, & \text { for } p>\frac{r}{q} \\ C_{\mathbb{V}}\left(|X|^{r / q} \ln ^{2}|X|\right)<\infty, & \text { for } p=\frac{r}{q} \\ C_{\mathbb{V}}\left(|X|^{r / q} \ln |X|\right)<\infty, & \text { for } p<\frac{r}{q}\end{cases}
$$

$$
\begin{aligned}
\int_{1}^{\infty} \mathrm{d} y \int_{1}^{\infty} y^{r-1} \ln (y) \mathbb{V}\left(|X|>x^{1 / p} y^{q}\right) \mathrm{d} x & \approx \int_{1}^{\infty} \mathrm{d} t \int_{t^{q}}^{\infty} t^{r-1} \ln (t) \mathbb{V}(|X|>s) s^{p-1} t^{-q p} \mathrm{~d} s\left(\operatorname{Setting}=x^{1 / p} y^{q}, t=y\right) \\
& \approx \int_{1}^{\infty} \mathbb{V}(|X|>s) s^{p-1} \mathrm{~d} s \int_{1}^{s^{1 / q}} t^{r-1-q p} \ln (t) \mathrm{d} t \\
& \approx \begin{cases}\int_{1}^{\infty} \mathbb{V}(|X|>s) s^{p-1} \mathrm{~d} s \approx C_{\mathbb{V}}\left(|X|^{p}\right), & \text { for } p>\frac{r}{q}, \\
\int_{1}^{\infty} \mathbb{V}(|X|>s) s^{r / q-1} \ln ^{2}(s) \mathrm{d} s \approx C_{\mathbb{V}}\left(|X|^{r / q} \ln ^{2}|X|\right), & \text { for } p=\frac{r}{q}, \\
\int_{1}^{\infty} \mathbb{V}(|X|>s) s^{p-1+(r-p q / q)} \ln (s) \mathrm{d} s \approx C_{\mathbb{V}}\left(|X|^{r / q} \ln |X|\right), & \text { for } p<\frac{r}{q}\end{cases}
\end{aligned}
$$

Proof

$$
\int_{1}^{\infty} \mathrm{d} y \int_{1}^{\infty} y^{r-1} \ln (y) \mathbb{V}\left(|X|>x^{1 / p} y^{q}\right) \mathrm{d} x<\infty .
$$




\section{Main Results}

We state our main results, the proofs of which will be given in Section 4

Theorem 1. Let $\left\{X_{n}, n \geq 1\right\}$ be a sequence of independent random variables, identically distributed as $X$ under sublinear expectation space $(\Omega, \mathscr{H}, \mathbb{E})$. Assume that $r>1$, $q>(1 / 2)$, and $\beta>-q / r$, and suppose that $\mathbb{E} X=-\mathbb{E}(-X)=0$ for $(1 / 2)<q \leq 1$. Suppose that $\left\{a_{n i} \approx(i / n)^{\beta} \quad\left(1 / n^{q}\right), 1\right.$ $\leq i \leq n, n \geq 1\}$ is a triangular array of real numbers. Then, the following is equivalent:

(i)

$$
\begin{cases}C_{\mathbb{V}}\left(|X|^{p}\right)<\infty, & \text { for } p>\frac{r}{q}, \\ C_{\mathbb{V}}\left(|X|^{r / q} \ln |X|\right)<\infty, & \text { for } p=\frac{r}{q}, \\ C_{\mathbb{V}}\left(|X|^{r / q}\right)<\infty, & \text { for } p<\frac{r}{q}\end{cases}
$$

(ii)

$$
\sum_{n=1}^{\infty} n^{r-2} C_{\mathbb{V}}\left(\left(\max _{1 \leq k \leq n}\left|\sum_{i=1}^{k} a_{n i} X_{i}\right|^{p}-\varepsilon\right)^{+}\right)<\infty, \quad \forall \varepsilon>0 .
$$

Remark 2. If $(\Omega, \mathscr{H}, \mathbb{E})$ is classic probability space, then Theorem 1 recovers Theorem 7 in [23] in case in which $\left\{X_{n}, n \geq 1\right\}$ is a sequence of independent random variables, identically distributed as $X$. As pointed in Hossein and Nezakati [27], why we need $p$ th moment convergence under sublinear expectations, the complete moment covergence is a more general expression than complete convergence in theory and practice, and the interested reader also could refer to the first study in complete moment convergence by Chow [28].

Remark 3. Under the same conditions in Theorem 1, if (28) holds, by the same proof of Remark 3.2 of Hossein and Nezakati [27], we see that, for all $\epsilon>0$,

$$
\sum_{n=1}^{\infty} n^{r-2} \mathbb{V}\left(\max _{1 \leq k \leq n}\left|\sum_{i=1}^{k} a_{n i} X_{i}\right|^{p}>\epsilon\right)<\infty
$$

Moreover, if $\mathbb{V}$ is continuous, by (29), we see that

$$
\sum_{i=1}^{n} a_{n i} X_{i} \longrightarrow 0, \quad \text { a.s. } \mathbb{V} \text {, as } n \longrightarrow \infty \text {. }
$$

In $(30)$, if $(1 / 2)<q \leq 1, \quad \beta=0$, we conclude that $\sum_{i=1}^{n} X_{i} / n^{q} \longrightarrow 0$ a.s. $\mathbb{V}$, as $n \longrightarrow \infty$, which is similar to Theorem 1 of Zhang and Lin [29].
Theorem 2. Let $\left\{X_{n}, n \geq 1\right\}$ be a sequence of independent random variables, identically distributed as $X$ under sublinear expectation space $(\Omega, \mathscr{H}, \mathbb{E})$. Assume that $r>1$, $q>(1 / 2)$, and $\beta=-q / r<0$, and suppose that $\mathbb{E} X=-\mathbb{E}(-X)=0$ for $(1 / 2)<q \leq 1$. Suppose that $\left\{a_{n i} \approx(i / n)^{\beta}\left(1 / n^{q}\right), 1 \leq i \leq n, n \geq 1\right\}$ is a triangular array of real numbers. Then, (28) is equivalent to

$$
\begin{cases}C_{\mathbb{V}}\left(|X|^{p}\right)<\infty, & \text { for } p>\frac{r}{q}, \\ C_{\mathbb{V}}\left(|X|^{r / q} \ln ^{2}|X|\right)<\infty, & \text { for } p=\frac{r}{q}, \\ C_{\mathbb{V}}\left(|X|^{r / q} \ln |X|\right)<\infty, & \text { for } p<\frac{r}{q} .\end{cases}
$$

Theorem 3. Let $\left\{X_{n}, n \geq 1\right\}$ be a sequence of independent random variables, identically distributed as $X$ under sublinear expectation space $(\Omega, \mathscr{H}, \mathbb{E})$. Assume that $r>1$, $q>(1 / 2)$, and $-q<\beta<-q / r<0$, and suppose that $\mathbb{E} X=-\mathbb{E}(-X)=0$ for $(1 / 2)<q \leq 1$. Suppose that $\left\{a_{n i} \approx(i / n)^{\beta}\left(1 / n^{q}\right), 1 \leq i \leq n, n \geq 1\right\}$ is a triangular array of real numbers. Then, (28) is equivalent to

$$
\begin{cases}C_{\mathbb{V}}\left(|X|^{p}\right)<\infty, & \text { for } p>\frac{(r-1)}{(q+\beta)} \\ C_{\mathbb{V}}\left(|X|^{(r-1) /(q+\beta)} \ln |X|\right)<\infty, & \text { for } p=\frac{(r-1)}{(q+\beta)}, \\ C_{\mathbb{V}}\left(|X|^{(r-1) /(q+\beta)}\right)<\infty, & \text { for } p<\frac{(r-1)}{(q+\beta)} .\end{cases}
$$

As in the proofs of Theorems 1-3, we can get the following corollary.

Corollary 1. Let $\left\{X_{n}, n \geq 1\right\}$ be a sequence of independent random variables, identically distributed as $X$ under sublinear expectation space $(\Omega, \mathscr{H}, \mathbb{E})$. Assume that $r>1$, $q>(1 / 2)$, and $\beta>-q$, and suppose that $\mathbb{E} X=-\mathbb{E}(-X)=0$, for $(1 / 2)<q \leq 1$. Suppose that $\left\{a_{n i} \approx((n-i) / n)^{\beta}\left(1 / n^{q}\right), 0\right.$ $\leq i \leq n-1, n \geq 1\}$ is a triangular array of real numbers. Then,

(i) (27) is equivalent to

$$
\sum_{n=1}^{\infty} n^{r-2} \mathbb{E}\left(\left(\max _{0 \leq k \leq n-1}\left|\sum_{i=0}^{k} a_{n i} X_{i}\right|^{p}-\epsilon\right)^{+}\right)<\infty, \quad \beta>\frac{-q}{r} .
$$

(ii) (31) is equivalent to (33) when $\beta=-q / r$.

(iii) (32) is equivalent to (33) when $-q<\beta<-q / r$. 
The following theorem is complete pth moment convergence on Cesàro summation of independent, identically distributed random variables under $(\Omega, \mathscr{H}, \mathbb{E})$.

Theorem 4. Let $\left\{X_{n}, n \geq 1\right\}$ be a sequence of independent random variables, identically distributed as $X$ under sublinear expectation space $(\Omega, \mathscr{H}, \mathbb{E})$. Assume that $r>1$, $q>(1 / 2), \quad 0<\alpha \leq 1, \quad$ and $p>0$, and suppose that $\mathbb{E} X=-\mathbb{E}(-X)=0, \quad$ for $\quad(1 / 2)<q \leq 1$. Suppose $A_{n}^{\alpha}=[(\alpha+1)(\alpha+2), \ldots,(\alpha+n)] / n !, \quad n=1,2, \ldots \quad$ and $A_{0}^{\alpha}=1$. Then,

(i) (27) is equivalent to

$$
\sum_{n=1}^{\infty} n^{r-2}\left(A_{n}^{\alpha}\right)^{-p} \mathbb{E}\left(\left(\max _{0 \leq k \leq n-1}\left|\sum_{i=0}^{k} A_{n-i}^{\alpha-1} X_{i}\right|^{p}-\epsilon\left(A_{n}^{\alpha}\right)^{p}\right)^{+}\right)<\infty, \quad \forall \epsilon>0,
$$

when $1-1 / r<\alpha \leq 1$.

(ii) (31) is equivalent to (34) when $\alpha=1-1 / r$.

(iii) (34) is equivalent to

$$
\begin{cases}C_{\mathbb{V}}\left(|X|^{p}\right)<\infty, & \text { for } p>\frac{(r-1)}{(q \alpha)}, \\ C_{\mathbb{V}}\left(|X|^{(r-1) /(q \alpha)} \ln |X|\right)<\infty, & \text { for } p=\frac{(r-1)}{(q \alpha)} \\ C_{\mathbb{V}}\left(|X|^{(r-1) /(q \alpha)}\right)<\infty, & \text { for } p<\frac{(r-1)}{(q \alpha)}\end{cases}
$$

when $0<\alpha<1-1 / r$.

Remark 4. If $(\Omega, \mathscr{H}, \mathbb{E})$ is classic probability space, then Theorems 1-3 and Corollary 1 recover, respectively, Theorems 10, 11, and 14 and Corollary 13 in Guo and Shan [23] in case in which $\left\{X_{n}, n \geq 1\right\}$ is a sequence of independent random variables, identically distributed as $X$.

\section{Proofs of the Main Results}

Proof of Theorem 1. We first prove that (27) implies (28). Notice that

$$
\begin{aligned}
& \sum_{n=1}^{\infty} n^{r-2} C_{\mathbb{V}}\left(\left(\max _{1 \leq k \leq n}\left|\sum_{i=1}^{k} a_{n i} X_{i}\right|^{p}-\epsilon\right)^{+}\right) \\
= & \sum_{n=1}^{\infty} n^{r-2} \int_{0}^{\infty} \mathbb{V}\left(\max _{1 \leq k \leq n}\left|\sum_{i=1}^{k} a_{n i} X_{i}\right|^{p}>\epsilon+x\right) \mathrm{d} x \\
= & \sum_{n=1}^{\infty} n^{r-2} \int_{\epsilon}^{1} \mathbb{V}\left(\max _{1 \leq k \leq n}\left|\sum_{i=1}^{k} a_{n i} X_{i}\right|^{p}>x\right) \mathrm{d} x+\sum_{n=1}^{\infty} n^{r-2} \int_{1}^{\infty} \mathbb{V}\left(\max _{1 \leq k \leq n}\left|\sum_{i=1}^{k} a_{n i} X_{i}\right|^{p}>x\right) \mathrm{d} x \\
\leq & \sum_{n=1}^{\infty} n^{r-2} \mathbb{V}\left(\max _{1 \leq k \leq n}\left|\sum_{i=1}^{k} a_{n i} X_{i}\right|>\epsilon^{1 / p}\right)+\sum_{n=1}^{\infty} n^{r-2} \int_{1}^{\infty} \mathbb{V}\left(\max _{1 \leq k \leq n}\left|\sum_{i=1}^{k} a_{n i} X_{i}\right|>x^{1 / p}\right) \mathrm{d} x \\
:= & \mathrm{I}+\mathrm{II} .
\end{aligned}
$$

From Xu and Cheng [24] and the fact that (27) implies $C_{\mathbb{V}}\left(|X|^{r / q}\right)<\infty$, we see that $\mathrm{I}<\infty$. We next establish II $<\infty$. Choose $0<\alpha<1 / p$, and $\delta>0$ is small sufficiently and integer $K$ is large enough. For every $1 \leq i \leq n, n \geq 1$, we note the fact that $n$ is large sufficiently to guarantee $x^{\alpha} n^{-\delta}<\left(x^{1 / p} / 4 K\right)$. Since the first finite terms of the series do not affect the convergence of the series, without loss of restictions, we could assume the definitions of $X_{n i}^{(j)}$ below are meaningful. Write 


$$
\begin{aligned}
& X_{n i}^{(1)}=-x^{\alpha} n^{-\delta} I\left(a_{n i} X_{i}<-x^{\alpha} n^{-\delta}\right)+a_{n i} X_{i} I\left(\left|a_{n i} X_{i}\right| \leq x^{\alpha} n^{-\delta}\right)+x^{\alpha} n^{-\delta} I\left(a_{n i} X_{i}>x^{\alpha} n^{-\delta}\right) \\
& X_{n i}^{(2)}=\left(a_{n i} X_{i}-x^{\alpha} n^{-\delta}\right) I\left(x^{\alpha} n^{-\delta}<a_{n i} X_{i}<\frac{x^{1 / p}}{4 K}\right) \\
& X_{n i}^{(3)}=\left(a_{n i} X_{i}+x^{\alpha} n^{-\delta}\right) I\left(-\frac{x^{1 / p}}{4 K}<a_{n i} X_{i}<-x^{\alpha} n^{-\delta}\right) \\
& X_{n i}^{(4)}=\left(a_{n i} X_{i}+x^{\alpha} n^{-\delta}\right) I\left(a_{n i} X_{i} \leq-\frac{x^{1 / p}}{4 K}\right)+\left(a_{n i} X_{i}-x^{\alpha} n^{-\delta}\right) I\left(a_{n i} X_{i} \geq \frac{x^{1 / p}}{4 K}\right)
\end{aligned}
$$

Observe that $\sum_{i=1}^{k} a_{n i} X_{i}=\sum_{i=1}^{k} X_{n i}^{(1)}+\sum_{i=1}^{k} X_{n i}^{(2)}+\sum_{i=1}^{k}$ $X_{n i}^{(3)}+\sum_{i=1}^{k} X_{n i}^{(4)}$. Notice that

$$
\left(\max _{1 \leq k \leq n}\left|\sum_{i=1}^{k} a_{n i} X_{i}\right|>x^{1 / p}\right) \subset \bigcup_{j=1}^{4}\left(\max _{1 \leq k \leq n}\left|\sum_{i=1}^{k} X_{n i}^{(j)}\right|>\frac{x^{1 / p}}{4}\right) .
$$

Consequently, to prove (28), we only need to prove that

$$
J_{j}:=\sum_{n=1}^{\infty} n^{r-2} \int_{1}^{\infty} \mathbb{V}\left(\max _{1 \leq k \leq n}\left|\sum_{i=1}^{k} X_{n i}^{(j)}\right|>\frac{x^{1 / p}}{4}\right) \mathrm{d} x<\infty, \quad j=1,2,3,4 .
$$

From the definition of $X_{n i}^{(4)}$, we deduce that

$$
\int_{1}^{s^{1 / q}} t^{\beta(r-1) /(q+\beta)} \mathrm{d} t \approx s^{(1 / q)+(\beta(r-1) / q(q+\beta))} .
$$

$$
\left(\max _{1 \leq k \leq n}\left|\sum_{i=1}^{k} X_{n i}^{(4)}\right|>\frac{x^{1 / p}}{4}\right) \subset\left(\max _{1 \leq i \leq n}\left|a_{n i} X_{n i}\right|>\frac{x^{1 / p}}{4 K}\right) \text {. }
$$

From $a_{n i} \approx(i / n)^{\beta}\left(1 / n^{q}\right)$, we see that

Observe that $\beta>-q / r$ implies $\beta(r-1) /(q+\beta)>-1$. Therefore,

$$
\begin{aligned}
J_{4} & \leq \sum_{n=1}^{\infty} n^{r-2} \sum_{i=1}^{n} \int_{1}^{\infty} \mathbb{V}\left(\left|a_{n i} X_{n i}\right|>\frac{x^{1 / p}}{4 K}\right) \mathrm{d} x \\
& \approx \sum_{n=1}^{\infty} n^{r-2} \sum_{i=1}^{n} \int_{1}^{\infty} \mathbb{V}\left(|X|>\frac{x^{1 / p}}{4 C K} n^{q+\beta} i^{-\beta}\right) \mathrm{d} x \\
& \approx \int_{1}^{\infty} \mathrm{d} x \int_{1}^{\infty} u^{r-2} \mathrm{~d} u \int_{1}^{u} \mathbb{V}\left(|X|>\frac{x^{1 / p}}{4 C K} u^{q+\beta} v^{-\beta}\right) \mathrm{d} v\left(\operatorname{Setting} s=u^{q+\beta} v^{-\beta}, t=v\right) \\
& \approx \int_{1}^{\infty} \mathrm{d} x \int_{1}^{\infty} \mathrm{d} s \int_{1}^{s^{1 / q}} s^{(r-1) /(q+\beta)-1} t^{\beta(r-1) /(q+\beta)} \mathbb{V}\left(|X|>\frac{x^{1 / p}}{4 C K} s\right) \mathrm{d} t \\
& \approx \int_{1}^{\infty} \mathrm{d} x \int_{1}^{\infty} s^{r / q-1} \mathbb{V}\left(|X|>\frac{x^{1 / p}}{4 C K} s\right) \mathrm{d} s .
\end{aligned}
$$

Hence, from Lemma 3 and (27), we obtain $J_{4}<\infty$. From the definition of $X_{n i}^{(2)}$, we conclude that $X_{n i}^{(2)} \geq 0$. From the subadditivity of capacity and Definition 2.5 in [10], it follows that 


$$
\begin{aligned}
& \mathbb{V}\left(\max _{1 \leq k \leq n}\left|\sum_{i=1}^{k} X_{n i}^{(2)}\right|>\frac{x^{1 / p}}{4}\right)=\mathbb{V}\left(\sum_{i=1}^{n} X_{n i}^{(2)}>\frac{x^{1 / p}}{4}\right) \\
\leq & \mathbb{V}\left(\text { there are at least } K \text { indices } i \in[1, n] \text { such that } a_{n i} X_{n i}>x^{\alpha} n^{-\delta}\right) \\
\leq & \sum_{1 \leq i_{1}<i_{2}<\cdots<i_{K} \leq n} \mathbb{V}\left(a_{n i_{j}} X_{i_{j}}>x^{\alpha} n^{-\delta}, \text { for all1 } \leq j \leq K\right) \\
( & \text { In Definition } \left.2.5 \text { in Chen }[10], \text { we set } X=\left(a_{n i_{1}} X_{i_{1}}, \ldots, a_{n i_{K-1}} X_{i_{K-1}}\right), Y=a_{n i_{K}} X_{i_{K}}\right) \\
( & \left.\varphi\left(X, Y_{n}\right)=I\left(a_{n i_{1}} X_{i_{1}}>x^{\alpha} n^{-\delta}, \ldots, a_{n i_{K-1}} X_{i_{K-1}}>x^{\alpha} n^{-\delta}, a_{n i_{K}} X_{i_{K}}>x^{\alpha} n^{-\delta}\right)\right) \\
= & \sum_{1 \leq i_{1}<i_{2}<\cdots<i_{K} \leq n} \mathbb{E}\left[I\left(a_{n i_{1}} X_{i_{1}}>x^{\alpha} n^{-\delta}, \ldots, a_{n i_{K-1}} X_{i_{K-1}}>x^{\alpha} n^{-\delta}\right)\right] \mathbb{V}\left(a_{n i_{K}} X_{i_{K}}>x^{\alpha} n^{-\delta}\right) \\
= & \cdots=\sum_{1 \leq i_{1}<i_{2}<\cdots<i_{K} \leq n} \prod_{j=1}^{K} \mathbb{V}\left(a_{n i_{j}} X_{i_{j}}>x^{\alpha} n^{-\delta}\right) \leq\left[\sum_{j=1}^{n} \mathbb{V}\left(a_{n j} X>x^{\alpha} n^{-\delta}\right)\right]^{K} .
\end{aligned}
$$

From $\quad \beta>-q / r, \quad$ we obtain $\sum_{i=1}^{n} a_{n i}^{r / q} \approx$ $\sum_{i=1}^{n} n^{-r(q+\beta) / q_{i} \beta r / q} \approx n^{1-r}$. Since (27) implies $\mathbb{E}|X|^{r / q}<\infty$, by Markov's inequality under sublinear expectations (cf. (9) in $\mathrm{Hu}$ and $\mathrm{Wu}[30])$ and (43), we conclude that

$$
\begin{aligned}
J_{2} & \leq \sum_{n=1}^{\infty} n^{r-2} \int_{1}^{\infty}\left[\sum_{j=1}^{n} \mathbb{V}\left(a_{n j} X>x^{\alpha} n^{-\delta}\right)\right]^{K} \mathrm{~d} x \\
& \leq \sum_{n=1}^{\infty} n^{r-2} \int_{1}^{\infty}\left(\sum_{j=1}^{n} x^{-r \alpha / q} n^{r \delta / q} a_{n j}^{r / q} \mathbb{E}|X|^{r / q}\right)^{K} \mathrm{~d} x \\
& \approx \sum_{n=1}^{\infty} n^{r-2-K(r-1-r \delta / q)} \int_{1}^{\infty} x^{-r K \alpha / q} \mathrm{~d} x .
\end{aligned}
$$

Notice that $r>1$ and $\alpha>0$; we could choose $\delta$ small sufficiently and integer $K$ large enough such that $r-2-$ $K(r-1-r \delta / q)<-1$ and $-r K \alpha / q<-1$. Hence, from (44), we obtain $J_{2}<\infty$. Similarly, we can get $J_{3}<\infty$. In order to estimate $J_{1}$, we first prove that

$$
\sup _{x \geq 1} \frac{1}{x^{1 / p}} \max _{1 \leq k \leq n}\left|\sum_{i=1}^{k} \mathbb{E} X_{n i}^{(1)}\right| \longrightarrow 0, \quad \text { as } n \longrightarrow \infty .
$$

Observe that (27), Lemma 4.5 in Zhang [4], and Hölder's inequality under sublinear expectations imply that $\mathbb{E}|X|^{r / q}<\infty$ and $\mathbb{E}|X|^{1 / p}<\infty$. When $q>1$, observe that $\left|X_{n i}^{(1)}\right| \leq x^{\alpha} n^{-\delta}$ and $\left|X_{n i}^{(1)}\right| \leq\left|a_{n i} X_{i}\right|$, by Hölder's inequality, we see that

$$
\begin{aligned}
\max _{1 \leq k \leq n}\left|\sum_{i=1}^{k} \mathbb{E} X_{n i}^{(1)}\right| & \leq \sum_{i=1}^{n} \mathbb{E}\left|X_{n i}^{(1)}\right| \leq x^{\alpha(1-1 / q)} n^{-\delta(1-1 / q)} \sum_{i=1}^{n} \mathbb{E}\left|a_{n i} X_{i}\right|^{1 / q} \\
& \ll x^{\alpha(1-1 / q)} n^{-\delta(1-1 / q)} \sum_{i=1}^{n}\left|a_{n i}\right|^{1 / q} \\
& \leq x^{\alpha(1-1 / q)} n^{-\delta(1-1 / q)} n^{(r-1) / r}\left(\sum_{i=1}^{n}\left|a_{n i}\right|^{r / q}\right)^{1 / r} \\
& \approx x^{\alpha(1-1 / q)} n^{-\delta(1-1 / q)} .
\end{aligned}
$$

Observing that $\alpha(1-1 / q)<\alpha<1 / p$, by (46), for any $x \geq 1$, we obtain

$$
\sup _{x \geq 1} \frac{1}{x^{1 / p}} \max _{1 \leq k \leq n}\left|\sum_{i=1}^{k} \mathbb{E} X_{n i}^{(1)}\right| \ll n^{-\delta(1-1 / q)} \longrightarrow 0, \quad \text { as } n \longrightarrow \infty \text {. }
$$


When $1 / 2<q \leq 1$, noticing that $\mathbb{E}(X)=\mathbb{E}(-X)=0$ and by choosing $\delta$ small sufficiently such that $-\delta(1-r / q)+1-r<0$, we see that

$$
\begin{aligned}
\max _{1 \leq k \leq n}\left|\sum_{i=1}^{k} \mathbb{E} X_{n i}^{(1)}\right| & \leq 2 \sum_{i=1}^{n} \mathbb{E}\left|a_{n i} X_{i}\right| I\left(\left|a_{n i} X_{i}\right|>x^{\alpha} n^{-\delta}\right) \\
& \leq 2 x^{\alpha(1-r / q)} n^{-\delta(1-r / q)} \sum_{i=1}^{n} \mathbb{E}\left|a_{n i} X_{i}\right|^{r / q} \\
& \leq 2 x^{\alpha(1-r / q)} n^{-\delta(1-r / q)} \sum_{i=1}^{n}\left|a_{n i}\right|^{r / q} \approx x^{\alpha(1-r / q)} n^{-\delta(1-r / q)+1-r} .
\end{aligned}
$$

Observing that $1-r / q<0$, we have

$\sup _{x \geq 1} \frac{1}{x^{1 / p}} \max _{1 \leq k \leq n}\left|\sum_{i=1}^{k} \mathbb{E} X_{n i}^{(1)}\right| \ll n^{-\delta(1-r / q)+1-r} \longrightarrow 0, \quad$ as $n \longrightarrow \infty$.

Thus, to establish $J_{1}<\infty$, we only need to prove that

$$
J_{1}^{*}:=\sum_{n=1}^{\infty} n^{r-2} \int_{1}^{\infty} \mathbb{V}\left(\max _{1 \leq k \leq n}\left|\sum_{i=1}^{k}\left(X_{n i}^{(1)}-\mathbb{E} X_{n i}^{(1)}\right)\right|>\frac{x^{1 / p}}{8}\right) \mathrm{d} x<\infty .
$$

Observe that $\left\{X_{n i}^{(1)}, 1 \leq i \leq n, n \geq 1\right\}$ is independent, identically distributed under $(\Omega, \mathscr{H}, \mathbb{E})$. By Markov's inequality under sublinear expectations, $C_{r}$ 's inequality, and
Lemma 1 , we conclude that, for a suitably large $M$, which will be determined later:

$$
\begin{aligned}
& \mathbb{V}\left(\max _{1 \leq k \leq n}\left|\sum_{i=1}^{k}\left(X_{n i}^{(1)}-\mathbb{E} X_{n i}^{(1)}\right)\right|>\frac{x^{1 / p}}{8}\right) \\
\ll & x^{-M / p}(\ln n)^{M}\left\{\sum_{i=1}^{n} \mathbb{E}\left|X_{n i}^{(1)}\right|^{M}+\left[\sum_{i=1}^{n} \mathbb{E}\left|X_{n i}^{(1)}\right|^{2}\right]^{M / 2}\right\} .
\end{aligned}
$$

Choosing large sufficiently $M$ such that $-M(1 / p-\alpha)-$ $r \alpha / q<-1$ and $-1-(M-r / q) \delta<-1$, we have

$$
\begin{aligned}
& \sum_{n=1}^{\infty} n^{r-2}(\ln n)^{M} \sum_{i=1}^{n} \int_{1}^{\infty} x^{-M / p} \mathbb{E}\left|X_{n i}^{(1)}\right|^{M} \mathrm{~d} x \\
\ll & \sum_{n=1}^{\infty} n^{r-2} n^{-\delta(M-r / q)}(\ln n)^{M} \sum_{i=1}^{n}\left|a_{n i}\right|^{r / q} \int_{1}^{\infty} x^{-M(1 / p-\alpha)-r \alpha / q} \mathrm{~d} x \\
\ll & \sum_{n=1}^{\infty} n^{-1-\delta(M-r / q)}(\ln n)^{M}<\infty .
\end{aligned}
$$

When $r / q \geq 2$, (27) implies that $\mathbb{E}|X|^{2}<\infty$. Observing $\quad-M / p<-1, \quad r-2-(2 q-1) M / 2<-1$. Then, by that $q>1 / 2$, we could choose $M$ large enough such that $\beta>-q / r \geq-(1 / 2)$,

$$
\begin{aligned}
& \sum_{n=1}^{\infty} n^{r-2}(\ln n)^{M} \int_{1}^{\infty} x^{-M / p}\left[\sum_{i=1}^{n} \mathbb{E}\left|X_{n i}^{(1)}\right|^{2}\right]^{M / 2} \mathrm{~d} x \\
\ll & \sum_{n=1}^{\infty} n^{r-2}(\ln n)^{M}\left(\sum_{i=1}^{n} a_{n i}^{2}\right)^{M / 2} \int_{1}^{\infty} x^{-M / p} \mathrm{~d} x \\
\ll & \sum_{n=1}^{\infty} n^{r-2}(\ln n)^{M}\left(\sum_{i=1}^{n} i^{2 \beta} n^{-2(q+\beta)}\right)^{M / 2} \\
\ll & \sum_{n=1}^{\infty} n^{r-2-(2 q-1) M / 2}(\ln n)^{M}<\infty .
\end{aligned}
$$


When $r / q<2$, choosing $M$ large enough such that $-M[1 / p-\alpha+\alpha r /(2 q)]<-1$ and $r-2-[\delta(2-r / q)+r-$ 1] $M / 2<-1$, we have

$$
\begin{aligned}
& \sum_{n=1}^{\infty} n^{r-2}(\ln n)^{M} \int_{1}^{\infty} x^{-M / p}\left[\sum_{i=1}^{n} \mathbb{E}\left|X_{n i}^{(1)}\right|^{2}\right]^{M / 2} \mathrm{~d} x \\
\ll & \sum_{n=1}^{\infty} n^{r-2} n^{-\delta(2-r / q) M / 2}(\ln n)^{M}\left(\sum_{i=1}^{n} a_{n i}^{r / q}\right)^{M / 2} \int_{1}^{\infty} x^{-M[1 / p-\alpha+\alpha r /(2 q)]} \mathrm{d} x \\
\ll & \sum_{n=1}^{\infty} n^{r-2-[\delta(2-r / q)+r-1] M / 2}(\ln n)^{M}<\infty .
\end{aligned}
$$

Consequently, by (51)-(54), we get $J_{1}^{*}<\infty$. Now, we prove that (28) implies (27). By Markov's inequality under sublinear expectations (see (8) of $\mathrm{Wu}[6]),(28)$, and Lemma 4.5 (iii) in [4],

$$
\begin{aligned}
& \sum_{n=1}^{\infty} n^{r-2} \mathbb{V}\left(\max _{1 \leq k \leq n}\left|\sum_{i=1}^{k} a_{n i} X_{i}\right|>\epsilon\right) \\
= & \sum_{n=1}^{\infty} n^{r-2} \mathbb{E}\left(1 \cdot I\left(\max _{1 \leq k \leq n}\left|\sum_{i=1}^{k} a_{n i} X_{i}\right|>\epsilon\right)\right) \\
\leq & \sum_{n=1}^{\infty} n^{r-2} \mathbb{E}\left(\left(\frac{\left(\max _{1 \leq k \leq n}\left|\sum_{i=1}^{k} a_{n i} X_{i}\right|^{p}-(\epsilon / 2)^{p}\right)^{+}}{(\epsilon / 2)^{p}}\right) I\left(\max _{1 \leq k \leq n}\left|\sum_{i=1}^{k} a_{n i} X_{i}\right|>\epsilon\right)\right) \\
\leq & \sum_{n=1}^{\infty} n^{r-2} \mathbb{E}\left(\left(\frac{\left(\max _{1 \leq k \leq n}\left|\sum_{i=1}^{k} a_{n i} X_{i}\right|^{p}-(\epsilon / 2)^{p}\right)^{+}}{(\epsilon / 2)^{p}}\right)\right) \\
\leq & \sum_{n=1}^{\infty} n^{r-2} \mathbb{E} \frac{\left(\max _{1 \leq k \leq n}\left|\sum_{i=1}^{k} a_{n i} X_{i}\right|^{p}-(\epsilon / 2)^{p}\right)^{+}}{(\epsilon / 2)^{p}}<\infty .
\end{aligned}
$$

Since

$$
\max _{1 \leq k \leq n}\left|a_{n k} X_{k}\right| \leq 2 \max _{1 \leq k \leq n}\left|\sum_{i=1}^{k} a_{n i} X_{i}\right|
$$

by (55) and similar proofs of (3.17) in [31], we conclude that

$$
\mathbb{V}\left(\max _{1 \leq k \leq n}\left|a_{n k} X_{k}\right|>\epsilon\right) \longrightarrow 0, \quad \text { as } n \longrightarrow \infty \text {. }
$$

Therefore, by (57) and Lemma 2, we obtain

$$
\sum_{i=1}^{n} \mathbb{V}\left(\left|a_{n i} X_{i}\right|>\epsilon\right) \ll \mathbb{V}\left(\max _{1 \leq k \leq n}\left|a_{n k} X_{k}\right|>\epsilon\right), \quad \text { for all } \epsilon>0 \text {. }
$$

Now, combining (58) with (28) gives

$$
\sum_{n=1}^{\infty} n^{r-2} \int_{\epsilon}^{\infty} \sum_{i=1}^{n} \mathbb{V}\left(\left|a_{n i} X_{i}\right|>x^{1 / p}\right) \mathrm{d} x<\infty .
$$

From the proof of (42), we conclude that (59) is equivalent to (27).

Proof. of Theorem 2. As in the proof of Theorem 1, with Lemma 4 in place of Lemma 3, and using

$$
\begin{gathered}
\sum_{i=1}^{n} a_{n i}^{r / q} \approx \sum_{i=1}^{n} n^{-r(q+\beta) / q_{i} \beta r / q} \approx n^{-r(q+\beta) / q / q} \ln n, \\
\int_{1}^{s^{1 / q}} t^{\beta(r-1) /(q+\beta)} \mathrm{d} t=\int_{1}^{s^{1 / q}} t^{-1} \mathrm{~d} t \approx \ln (s),
\end{gathered}
$$

when $\beta=-q / r$, we could prove Theorem 2 . Hence, the proof is omitted. 
Proof. of Theorem 3. As in the proof of Theorem 1, using

$$
\sum_{i=1}^{n} a_{n i}^{r / q} \approx \sum_{i=1}^{n} n^{-r(q+\beta) / q_{i} \beta r / q} \approx n^{-r(q+\beta) / q}, \quad \text { if } \beta<\frac{-q}{r},
$$

and with

$$
\int_{1}^{s^{1 / q}} t^{\beta(r-1) /(q+\beta)} \mathrm{d} t \approx C, \quad \text { if } \beta<\frac{-q}{r},
$$

in place of (41), we could prove Theorem 3. Thus, the proof is omitted.

Proof. of Theorem 4. Setting $a_{n i}=\left(A_{n-i}^{\alpha-1} / A_{n}^{\alpha}\right)^{q}, 0 \leq i \leq n$ and $n \geq 1$, we observe that $a_{n i} \approx(n-i)^{q(\alpha-1)} n^{-q \alpha}, 0 \leq i<n, n \geq 1$, and $a_{n n} \approx n^{-q \alpha}$. As in the proof of Theorem 14 in [23], letting $\beta=q(\alpha-1)$ in Corollary 1, we finish the proof of Theorem 4 .

\section{Data Availability}

No data were used to support this study.

\section{Conflicts of Interest}

The authors declare that they have no conflicts of interest.

\section{Authors' Contributions}

All authors contributed equally and read and approved the final manuscript.

\section{Acknowledgments}

This research was supported by Doctoral Scientific Research Starting Foundation of Jingdezhen Ceramic University (no. 102/01003002031), Scientific Program of Department of Education of Jiangxi Province of China (nos. GJJ190732 and GJJ180737), Natural Science Foundation Program of Jiangxi Province (no. 20202BABL211005), and National Natural Science Foundation of China (no. 61662037).

\section{References}

[1] S. Peng, "G-expectation, G-brownian motion and related stochastic calculus of itô type," Stochastic Analysis and Applications, vol. 2, no. 4, pp. 541-567, 2007.

[2] S. G. Peng, "Nonlinear expectations and stochastic calculus under uncertainty," 2010, https://arxiv.org/abs/1002.4546.

[3] L.-X. Zhang, "Donsker's invariance principle under the sublinear expectation with an application to chung;s law of the iterated logarithm," Communications in Mathematics and Statistics, vol. 3, no. 2, pp. 187-214, 2015.

[4] L. Zhang, "Exponential inequalities under the sub-linear expectations with applications to laws of the iterated logarithm," Science China Mathematics, vol. 59, no. 12, pp. 2503-2526, 2016a.

[5] L. X. Zhang, "Rosenthal's inequalities for independent and negatively dependent random variables under sub-linear expectations with applications," Science China Mathematics, vol. 59, no. 4, pp. 759-768, 2016b.

[6] Q. Y. Wu, "Precise asymptotics for complete integral convergence under sublinear expectations," Mathematical
Problems in Engineering, vol. 2020, Article ID 3145935, 13 pages, 2020.

[7] M. Z. Xu and K. Cheng, "Precise asymptotics in the law of the iterated logarithm under sublinear expectations," Mathematical Problems in Engineering, vol. 2021, Article ID 6691857, 9 pages, 2021.

[8] J. P. Xu and L. X. Zhang, "Three series theorem for independent random variables under sub-linear expectations with applications," Acta Mathematica Sinica, English Series, vol. 35, no. 2, pp. 172-184, 2019.

[9] J.-P. Xu and L.-X. Zhang, "The law of logarithm for arrays of random variables under sub-linear expectations," Acta Mathematicae Applicatae Sinica, English Series, vol. 36, no. 3, pp. 670-688, 2020.

[10] Z. Chen, "Strong laws of large numbers for sub-linear expectations," Science China Mathematics, vol. 59, no. 5, pp. 945-954, 2016.

[11] F. Q. Gao and M. Z. Xu, "Large deviations and moderate deviations for independent random variables under sublinear expectations (in Chinese)," Science China Mathematics, vol. 41, no. 4, pp. 337-352, 2011.

[12] X. Fang, S. G. Peng, Q. M. Shao, and Y. S. Song, "Limit theorems with rate of convergence under sublinear expectations," Bernoulli, vol. 25, no. 4A, pp. 2564-2596, 2018.

[13] F. Hu, Z. J. Chen, and D. F. Zhang, "How big are the increments of G-Brownian motion," Science China Mathematics, vol. 57, no. 8, pp. 1686-1700, 2014.

[14] Z.-C. Hu and Y.-Z. Yang, "Some inequalities and limit theorems under sublinear expectations," Acta Mathematicae Applicatae Sinica, English Series, vol. 33, no. 2, pp. 451-462, 2017.

[15] W. H. Huang and P. Y. Wu, "Strong laws of large numbers for general random variables in sublinear expectation spaces," Journal of Inequalities and Applications, vol. 2019, Article ID 143, 18 pages, 2019.

[16] A. Kuczmaszewska, "Complete convergence for widely acceptable random variables under the sublinear expecations," Journal of Mathematical Analysis and Applications, vol. 484, no. 1, p. 15, Article ID 123662, 2020.

[17] X. C. Ma and Q. Y. Wu, "Limiting behavior of weighted sums of extended negatively dependent random variables under sublinear expectations," Advances in Mathematics, vol. 48, no. 2, pp. 89-96, 2018.

[18] W. J. Wang and Q. Y. Wu, "Almost sure convergence of weighted sums for extended negatively dependent random variables under sub-linear expectations," Mathematica Applicata, vol. 32, no. 2, pp. 382-391, 2019.

[19] Q. Wu and Y. Jiang, "Strong law of large numbers and Chover's law of the iterated logarithm under sub-linear expectations," Journal of Mathematical Analysis and Applications, vol. 460, no. 1, pp. 252-270, 2018.

[20] D. L. Yu and Q. Y. Wu, "Marcinkiewicz type complete convergence for weighted sums under sub-linear expectations," Journal of University of Science and Technology of China, vol. 48, no. 2, pp. 89-96, 2018.

[21] L. X. Zhang, "Strong limit theorems for extended independent and extended negatively random variables under non-linear expectations," 2016, https://arxiv.org/abs/1608.0071v1.

[22] H. Y. Zhong and Q. Y. Wu, "Complete convergence and complete moment convergence for weighted sums of extended negatively dependent random variables under sublinear expectation," Journal of Inequalities and Applications, vol. 2017, Article ID 261, 14 pages, 2017. 
[23] M. L. Guo and S. W. Shan, "Equivalent conditions of complete $q$ th monent convergence for weighted sums of sequences of negatively orthant dependent raodom variables," Chinese Journal of Applied Probabability and Statistics, vol. 36, no. 4, pp. 381-392, 2020.

[24] M. Z. Xu and K. Cheng, "Equivalent conditions of complete convergence for weighted sums of sequences of i. i. d. random variables under sublinear expectations," 2021, https://arxiv. org/abs/2108.12085v1.

[25] F. Móricz, "Moment inequalities and the strong law of large numbers," Zeitschrift für Wahrscheinlichkeitstheorie und Verwandte Gebiete, vol. 35, no. 4, pp. 299-314, 1976.

[26] M. Z. Xu and K. Cheng, "Convergence for sums of independent, identically distributed random variables under sublinear expectations," 2021, https://arxiv.org/abs/2104. $10430 \mathrm{v} 1$.

[27] S. m. Hossenni and A. Nezakati, "Complete monent convergence for the dependent linear processes with random coefficients," Acta Mathematica Sinica, English Series, vol. 35, no. 8, pp. 113-132, 2019.

[28] Y. S. Chow, "On the rate of moment convergence of sample sums and extremes," Bulletin of the Institute of Mathematics Academia Sinica, vol. 16, no. 3, pp. 177-201, 1988.

[29] L. Zhang and J. Lin, "Marcinkiewicz's strong law of large numbers for nonlinear expectations," Statistics \& Probability Letters, vol. 137, pp. 269-276, 2018.

[30] R. Hu and Q. Y. Wu, "Complete convergence for weighted sums of widely acceptable random variables under sublinear expectations," Discrete Dynamics in Nature and Society, vol. 2021, Article ID 5526609, 10 pages, 2021.

[31] M. L. Guo, "Equivalent conditions of complete convergence for weighted sums of sequences of negatively dependent raodom variables," Abstract and Applied Analysis, vol. 2012, Article ID 425969, 13 pages, 2012. 